\title{
WAVELENGTH SELECTIVE DETECTION USING EXCITONIC RESONANCES IN GaAs/AIGaAs P-I-(MQW)-N STRUCTURES
}

\author{
Subrata Goswami, Pallab Bhattacharya, and Jasprit Singh \\ Center for High-Frequency Microelectronics \\ Department of Electrical Engineering and Computer Science \\ The University of Michigan, Ann Arbor, Michigan 48109-2122 \\ (Received $30 \mathrm{July}, 1990$ )
}

\begin{abstract}
The quantum confined Stark effect causes a strong wavelength and voltage dependence of photocurrent near excitonic resonances which is used to study the wavelength selectivity of $\mathrm{p}-\mathrm{i}(\mathrm{MQW})-\mathrm{n}$ photodiode. For a parallel input of optical bits each coming at a different wavelength, the selectivity is considered good if the state of a $\lambda_{i}$ wavelength bit can be detected regardless of the $\lambda_{j}(j \neq i)$ state of the bits. Photocurrent is found to have very good selectivity if $\lambda_{j}$ bits are all zero, i.e. the optical information is serial. However, we find that differential photocurrent $\left(\Delta I_{p h} / \Delta V\right)$ provides a good selectivity for random states of $\lambda_{j}$ bits (i.e. parallel input). Four channel selectivity is demonstrated at $200 \mathrm{~K}$. Specially designed quantum well structures can greatly improve this selectivity.
\end{abstract}

Fiber optic communication systems offer the possibility of very large bandwidth allowing information to be carried in many different wavelengths, without interference. Unfortunately, this capability is very difficult to utilize since most detection schemes can not accurately decode a particular wavelength when information is also randomly coming in at other wavelengths. Approaches to solve this difficulty involve techniques where selected wavelengths are allowed to transmit (using various tuned filters) while others are blocked, or through use of more complex coherent detection schemes $^{(1)}$. While these approaches are certainly important, the technology will greatly advance if the detectors themselves display wavelength selectivity. The optical detectors such as a photodiode is not expected to display wavelength selectivity. In most applications the tunable range of the energy of the photons would be $\leq 100 \mathrm{meV}$, where most detectors have a uniform response.

The photocurrent versus voltage characteristic of a reverse biased $\mathrm{p}-\mathrm{i}(\mathrm{MQW})-\mathrm{n}$ structure is known to exhibit strong wavelength dependence ${ }^{(2,3)}$ due to the heavy hole (HH) and light hole (LH) excitonic peaks and their dependence on electric field - the quantum confined Stark effect (QCSE) ${ }^{(4)}$. This dependence can be exploited to develop tunable detectors. Such a scheme works quite well, as we shall discuss below, if the optical information comes in serially at different wave- lengths. However, if the information comes in parallel, the photocurrent is unable to unravel the information contained in a given wavelength. We discuss in this letter a scheme to carry out selective detection with information impinging randomly in parallel.

$P-i-n$ detectors for our experiments were grown by molecular beam epitaxy (MBE). The i-region consists of 100 periods of multiquantum well consisting of alternate layers of $100 \AA \mathrm{GaAs}$ and $100 \AA \mathrm{Al}_{0.3} \mathrm{Ga}_{0.7}$ As. 500 $\mu \mathrm{m}$ diameter mesa diodes are defined by photolithography and appropriate ohmic contacts are formed on the $p$ - and $n$-sides by evaporation and annealing. The wavelength dependence of the photocurrent-voltage characteristics was measured by using the output of a tunable dye laser in the range $800-870 \mathrm{~nm}$ and a HP 4145 parameter analyzer.

In Fig. 1(a), we show the room temperature reverse biased photocurrent of the $p-i(M Q W)-n$ diode for a series of wavelengths of equal intensity. As can be clearly seen, even at room temperature, at a given bias value the photocurrent is quite distinct for different wavelengths. Using this structure with an external series resistance (load line shown in Fig. 1(a)) we see that the voltage across the $p-i-n$ structure has a strong wavelength dependence. Results in Fig. 1(b) are shown for $200 \mathrm{~K}$. At room temperature, a change in input wavelength of $100 \AA$ produces a change in the output reference voltage of $\sim 10 \mathrm{~V}$. If the input information is 




(a)

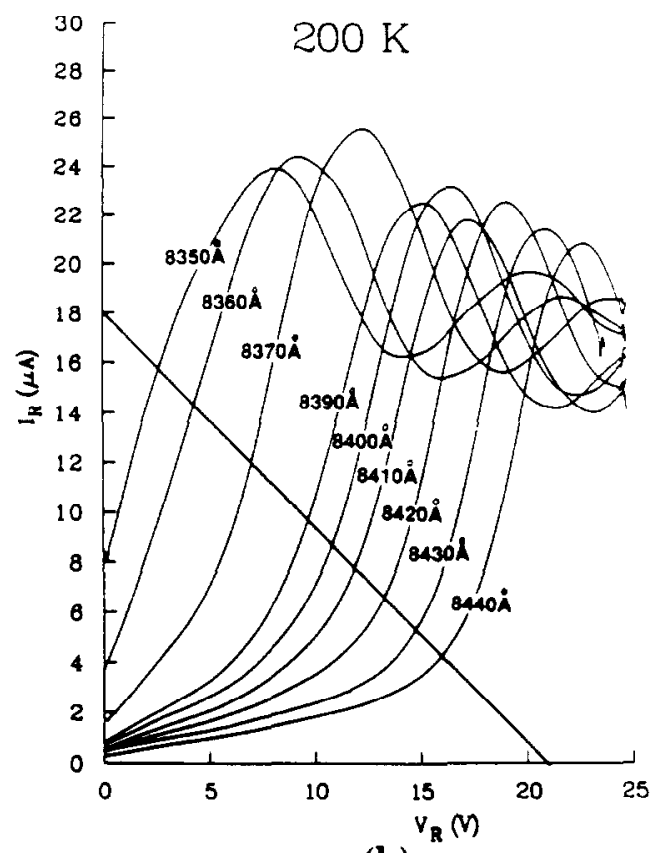

(b)

Figure 1 Measured photocurrent-voltage characteristics at various wavelengths in a $\mathrm{p}-\mathrm{i}(\mathrm{MQW})-\mathrm{n}$ photodiode (a) at $300 \mathrm{~K}$ and (b) at $200 \mathrm{~K}$.

coming serially, the output reference voltage in the circuit provides a very selective determination of the state (ON or OFF) of the chosen wavelength. This scheme will allow one to detect up to 50 wavelength channels serially, allowing a reference voltage across the resistor to vary by $\sim 0.2 \mathrm{~V}$.

While the serial detection of a given wavelength may be useful for many applications, in communication, one would like the detectors to selectively detect information in a channel at wavelength $\lambda_{i}$ regardless of the state of the channel at wavelength $\lambda_{j}(j \neq i)$. The photocurrents shown in Figs. 1(a) and 1(b) are not capable of doing this as can be easily seen by summing various $\lambda_{i}$ curves. However, if two $p-i-n$ structures are biased at voltages $V_{i}$ and $V_{i}+\Delta V_{i}$, a simple subtraction of the currents provides the value of $\frac{\Delta I \text { ph }}{\Delta V}$. In Fig. 2 we show this scheme of operation. To study the "parallel" selectivity of this device we show in Figs. 3(a)-(d), the values of $\frac{\Delta I p h}{\Delta V}$ at $200 \mathrm{~K}$ as a function of voltage, when information is coming in through four channels at wavelengths $8350 \AA, 8370 \AA, 8410 \AA$ and $8430 \AA$. The light curves in each diagram, for a selected $\lambda_{i}$, represent the results of $\sum_{i \neq i} \frac{\Delta I_{\mathrm{sh}}\left(\lambda_{i}\right)}{\Delta V}$ for all possible $\left(2^{\mathrm{s}}\right.$ -1) combination states (i.e. ON or OFF) of the other

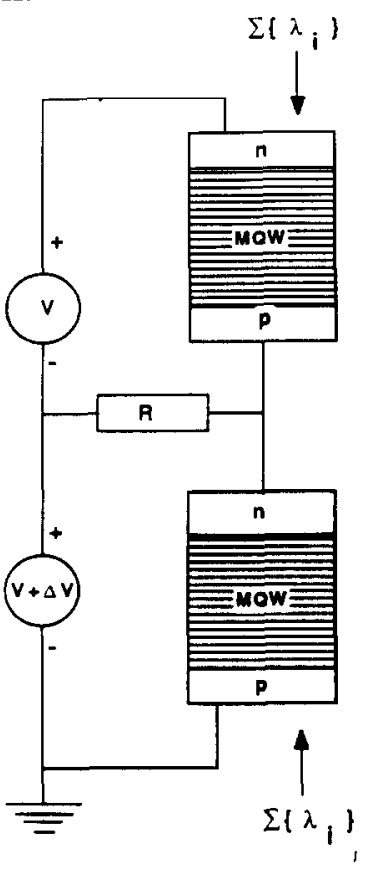

Figure 2

Schematic for photocurrent subtraction using two diodes for wavelength selective detection. The potential developed across the resistance $R$ is proportional to the differential photocurrent. 


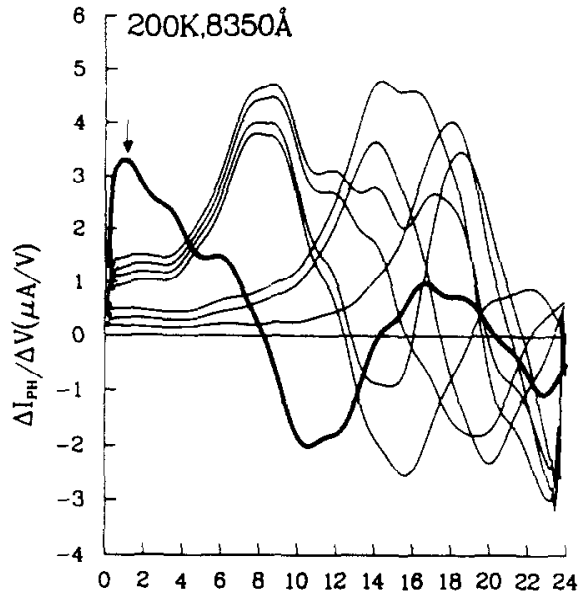

Reverse Bias(V)

(a)



Reverse Bias(V)

(c)

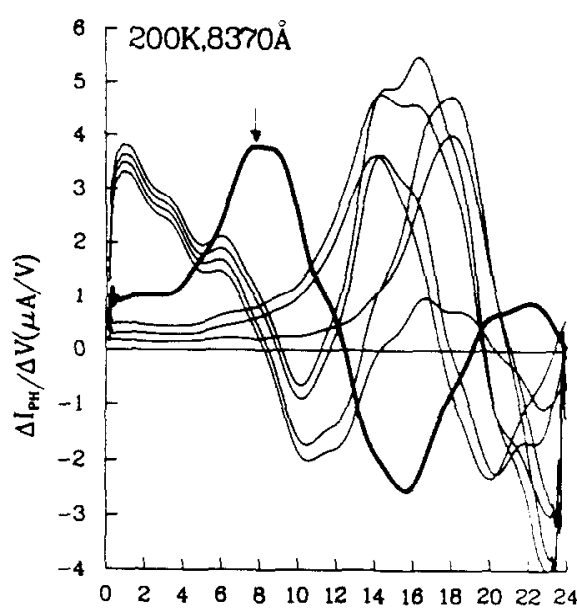

Reverse $\operatorname{Bias}(\mathrm{V})$

(b)

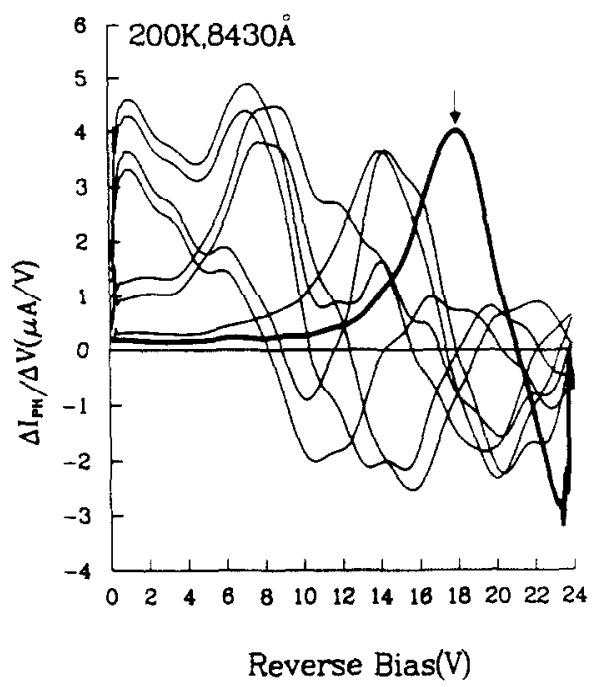

(d)

Figure 3 Values of $\Delta \mathrm{I}_{p h} / \Delta \mathrm{V}$ at $200 \mathrm{~K}$ for different states of 4 channels $\lambda_{j}$. The bold current represents the ON state of $\lambda_{i}$ only when $\lambda_{1}=8350 \AA(\mathrm{a}), 8370 \AA$ (b), $8410 \AA$ (c), and $8430 \AA$ (d).

$\left(\lambda_{j} \neq \lambda_{i}\right)$ channels. The bold line represents the value of $\frac{\Delta I_{\text {oh }}\left(\lambda_{i}\right)}{\Delta V}$ for the case where only channel $\lambda_{i}$ in ON.

From the results of Fig. 3, we see that if the difference current obtained from two $p-i(M Q W)-n$ diodes of Fig. 2 biased at values $1 \mathrm{~V}$ apart is compared with a constant current source of appropriate value $(2 \mu \mathrm{A}$ for our results) the state of the channel $\lambda_{i}$ can be detected regardless of the state of the other channels, if the devices are biased in the region indicated by the arrow. For example, in Fig. 3(a) shown for $\lambda_{i}=8350 \AA$, if the diodes are biased at $2.0 \pm 0.5 \mathrm{~V}$, the difference current is more than $2 \mu \mathrm{A}$ if the $\lambda_{i}$ bit is ON and less than 2 $\mu \mathrm{A}$ if it is OFF regardless of the states of the other channels. The same holds for $8370 \AA$ if the diodes are 


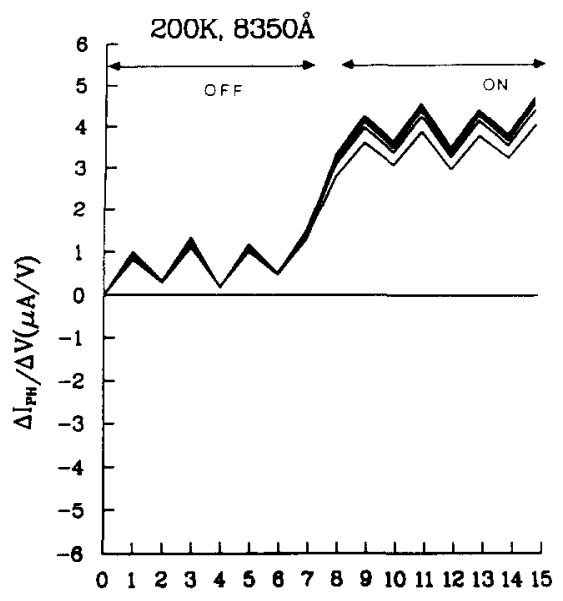

Figure 4

Channel

Values of $\Delta \mathrm{I}_{p h} / \Delta \mathrm{V}$ at $200 \mathrm{~K}$ plotted against the binary word representation of all four wavelength channels. The most significant wavelength here is $\lambda_{i}=8350 \AA$.

biased at $9.0 \pm 0.5 \mathrm{~V}$ and for $8410 \AA$ at $14.0 \pm 0.5 \mathrm{~V}$ and for $8430 \AA$ at $18.0+0.5 \mathrm{~V}$. The results are represented in a different way in Fig. 4. Considering the case of four incoming wavelength channels, each channel is assigned a digit in a binary four-bit word, the most significant bit being the wavelength $\lambda_{i}$ which needs to be detected. For the other three wavelengths $\lambda_{j}\left(\neq \lambda_{i}\right)$ a bit in the word is 1 if that particular $\lambda_{j}$ is $\mathrm{ON}$ and 0 if it is OFF. The abscissa represents the binary state of the channels which are $2^{4}$ in number. Hence, whenever $\lambda_{i}$ is ON, the channel number is greater than 7. Results in Fig. 4 are shown for $\lambda_{i}=8350 \AA$ with the diodes biased at $2.0 \pm 0.5 \mathrm{~V}$. The vertical axis is the differential change in photocurrent. The different curves in the diagram correspond to different biasing voltages around the optimum value as indicated in the plots of Fig. 2. It is clear that within a small incremental range of volt$\operatorname{agcs}( \pm 0.5 \mathrm{~V})$ the differential photocurrent jumps from
$1.5 \mu \mathrm{A} / \mathrm{V}$ to $4 \mu \mathrm{A} / \mathrm{V}$ as the most significant channel $\lambda_{i}$ is turned ON. Similar results are obtained for other values of $\lambda_{i}$.

From our studies we find that with operation at 200 $K$ four channel parallel information can be easily decoded. Also examining the value of the photoresponse, we see that an important source of non-selectivity in this scheme comes from the negative resistance region of the I-V response. If this region were not present, the selectivity is estimated to increase to $\sim 30$ channels. We are now pursuing the fabrication of $p-i(M Q W)-r$ structures where the i-region consists of multiquantum wells with two different well sizes so as to eliminate the negative resistance region and allow decoding of a layer number of channels. In summary we have shown that while the photocurrent in a $\mathrm{p}-\mathrm{i}(\mathrm{MQW})$-n structure has a very high wavelength selectivity, it can operate usefully only if the optical information is impinging serially. In that case by preadjusting a comparator voltage, the presence or absence of the given wavelength signal can be efficiently detected. If however, the information is impinging in parallel, differential photocurrent is more useful. A simple scheme proposed by us can carry out efficient decoding when information is coming in four wavelengths simultaneously.

Acknowledgement - This work was supported by a grant from the Air Force Office of Scientific Research under contract AFOSR-88-0168.

\section{References:}

1. Y. Yamamoto and T. Kimura, IEEE J. Quantum Electron. QE-17, 919 (1981).

2. J. Singh, S-C. Hong, P. K. Bhattacharya and R. Sahai, Appl. Optics, 27, 4554 (1988).

3. A. Larsson, P. A. Andrekson, S. T. Eng and A. Yariv, IEEE J. Quantum Electron. 24, 787 (1988).

4. D.A.B. Miller, D. S. Chemla, T. C. Damen, A. C. Gossard, W. Wiegmann, T. H. Wood and C. A. Burrus, Phys. Rev. Lett., 53, 2173 (1984). 